\title{
Effect of BMI and Binge Eating on Food Reward and Energy Intake: Further Evidence for a Binge Eating Subtype of Obesity
}

\author{
Michelle Dalton John Blundell Graham Finlayson \\ Institute of Psychological Sciences, Faculty of Medicine and Health, University of Leeds, Leeds, UK
}

\section{Key Words}

Hedonic eating · Food reward · Binge eating trait · Implicit wanting · Explicit liking

\begin{abstract}
Background: The psychological characteristics of binge eating have been proposed as a phenotype to further understanding of overconsumption and susceptibility to obesity. This study examined the influence of trait binge eating in lean and overweight or obese women on appetite, food reward and energy intake. Methods: 25 lean and 25 overweight or obese women were categorised as either 'binge type' or 'non-binge type' based on their scores on the Binge Eating Scale. Food reward and food intake were assessed in fasted and fed conditions. Results: Overweight or obese binge types (O-B) consumed more energy than overweight or obese non-binge types (O-NB) and lean binge (L-B) and non-binge types (L-NB). Both L-B and O-B exhibited greater preference for sweet foods. In O-NB, L-B and L-NB, lower liking and wanting for sweet foods was exhibited in the fed condition compared to the fasted condition. However, in O-B wanting for sweet foods was greater when they were fed compared to when they were in a fasted state. Conclusions: These findings provide further support for trait binge eating as a hedonic subtype of obesity. Binge types were characterised by greater intake of high-fat sweet foods and increased wanting for these foods when satiated. Additionally, these findings highlight the potential for separation in liking and wanting for food as a marker of susceptibility to overeat.
\end{abstract}

(c) 2013 S. Karger GmbH, Freiburg

\section{Introduction}

Overconsumption leading to a positive energy balance is a major determinant of weight gain and obesity. Under laboratory conditions, overweight and obese individuals have been shown to consume more energy in ad libitum eating tasks compared to their lean counterparts, even when hunger is suppressed [1-3]. Indeed, eating in a state of low (or absent) hunger appears to be a distinct risk factor for overconsumption. Therefore, it is important to 
Dalton et al.: Effect of BMI and Binge Eating on Food Reward and Energy Intake:

Further Evidence for a Binge Eating Subtype of Obesity

consider the mechanisms of eating behaviour in the presence and absence of hunger. Furthermore, within the normal-weight population a number of psychometric traits appear to reliably predict intake of highly palatable food across a wide range of experimental contexts [4-8]. This relative overconsumption also appears to be independent of any corresponding difference in sensations of appetite [9].

Food choice and energy intake are often determined by the expectation or experience of pleasure which can be understood in terms of wanting versus liking for food. In human appetite research, liking and wanting are often studied in relation to subjective states that refer to the everyday understanding of these terms. Wanting may describe subjective states of desire, while liking is characteristically understood as the appreciation of a foods sensory properties. Furthermore, liking and wanting for food are not necessarily consciously monitored or accessible to the individual. While people tend to be very good at estimating and reporting their liking for food, they are often unable to accurately gauge their implicit wanting for food (i.e. why they are automatically drawn to one food over another). A review of the literature on liking and wanting suggests that reward-driven eating is characterised by instances where liking and wanting become enhanced or even dissociated and therefore contribute to certain forms of overeating and eating pathology [10]. Indeed, reward-driven eating appears to counteract the effects of satiety [12] and leads to weight gain [13].

It is unlikely that all obese individuals are characterised by dysregulated food reward, but reward-based risk factors may help to characterise distinct phenotypes that exist within the obese population [14]. Such phenotypes can be examined by measuring liking and implicit (covert) wanting, that when enhanced may lead to greater intake of these foods.

It has been noted that patients with binge eating disorder display patterns of behaviour under experimental conditions that resemble enhanced food wanting [10]. Furthermore, the tendency to binge eat (as a non-clinical phenomenon), has been proposed as a plausible phenotype of obesity $[14,15]$. Recurrent episodes of binge eating are estimated to occur in $10-20 \%$ of obese individuals $[16,17]$ and constitute a trait that can be assessed psychometrically and applied to the general population. Importantly, the trait of binge eating occurs in normal-weight, overweight and obese individuals. In a study of non-obese women who varied in their tendency to binge eat according to the Binge Eating Scale (BES) [18], those with high scores showed a greater intake of high-fat sweet food, compared to those with low scores. In accordance with this finding, those with high scores showed an enhanced implicit wanting for high-fat sweet food combinations, as assessed by a computer-based procedure [4].

The current study sought to extend previous findings on food reward and food intake by examining individual differences in liking and implicit wanting in relation to both trait binge eating and obesity. Overweight and obese women were age-matched to normal-weight controls and subsequently identified as 'binge type' or 'non binge type' eaters. Food choice and ad libitum food intake along with measures of liking and implicit wanting using a computerised procedure were assessed in fasted or fed states. The study examined the hypothesis that obese 'binge types' would consume more food and display higher liking and implicit wanting for high-fat sweet foods according to their degree of hunger. The issue was whether or not the state of satiety carries a risk for overeating in obese individuals characterised as binge eaters.

\section{Participants and Methods}

\section{Participants}

25 overweight or obese (age: $25.3 \pm 8.9$ years, BMI: $30.7 \pm 3.4 \mathrm{~kg} / \mathrm{m}^{2}$ ) and 25 lean (age: $27.2 \pm 8.3$ years, BMI: $22 \pm 1.4 \mathrm{~kg} / \mathrm{m}^{2}$ ) females were recruited from the staff and student population at the University of Leeds, UK. Participants were selected from an initial screening process to exclude those who were taking medi- 
Dalton et al.: Effect of BMI and Binge Eating on Food Reward and Energy Intake:

Further Evidence for a Binge Eating Subtype of Obesity

cation, currently dieting, reported a history of eating disorders, or were unfamiliar with or disliked the study foods. Participants with a BMI between 18.5 and $24.9 \mathrm{~kg} / \mathrm{m}^{2}$ were classified as lean, and participants with a BMI above $27.5 \mathrm{~kg} / \mathrm{m}^{2}$ were classified as overweight or obese. Group status was verified by measures of waist circumference [19]. The groups were individually matched by age, with an overall difference of no more than 3 years across each matched pair. Informed written consent was obtained prior to the study. Participants received GBP 8.00 for their participation. All research procedures were reviewed and approved by the University of Leeds, Institute of Psychological Sciences Ethics Committee in accordance with the Helsinki declaration.

\section{Design}

The study conformed to a between-subjects crossover design with participants randomised to fasted or fed state conditions over two counter-balanced test sessions, separated by a minimum of 7 days. Participants were asked to refrain from eating or drinking anything besides water for $3.5 \mathrm{~h}$ after consuming their normal breakfast before the start of the experiment. Participants were also required to attend one morning screening session for which they were required to fast from 10 p.m. the evening before so that accurate measurements of height, weight and waist circumference could be taken. This session was held a minimum of 7 days before the start of the experiment and allowed for the test meal to be calibrated according to individual energy requirements.

\section{Measures}

Trait Binge Eating

Participants completed the BES [18] at the end of the experimental procedures. The scale is comprised of sixteen items, eight describing the behavioural manifestations and eight describing the feelings and cognitions associated with binge eating. Each item consists of three to four descriptive statements that increase in severity (e.g. 'I don't have any difficulty eating slowly in the proper manner' to 'I have the habit of bolting down my food without really chewing it. When this happens I usually feel uncomfortably stuffed because I've eaten too much'). Participants are required to select which statement from each of the sixteen items is most descriptive of them. Scores are summed to produce a total score ranging from $0-46$. Cut-off points have previously been reported denoting mild $(\leq 17)$, moderate (18-26) and severe $(\geq 27)$ binge eating behaviour [20]. The BES has been shown to have good internal validity, with a Cronbach's alpha of 0.89 [21] and good testretest reliability [22].

\section{Anthropometrics}

During the screening session, standing height without shoes was measured to the nearest $0.5 \mathrm{~cm}$ using a stadiometer. Body weight was measured using an electronic balance and recorded to the nearest $0.1 \mathrm{~kg}$. Waist circumference $(\mathrm{cm})$ was measured $1 \mathrm{~cm}$ above the top of the participants' naval after expiration.

\section{Subjective Appetite Sensations}

Subjective appetite sensations were measured using 100-mm visual analogue scales (VAS). Measures of hunger ('how hungry do you feel now?') and fullness ('how full do you feel right now?') were anchored at each end with the statements 'extremely' and 'not at all.' Ratings of prospective consumption ('how much food could you eat right now?') and desire to eat ('how strong is your desire to eat?') were anchored at each end by 'none at all' and 'a very large amount' and 'not very strong' and 'very strong', respectively. VAS have been shown be sensitive to experimental manipulations and have good test-retest reliability [23].

\section{Food Reward: Explicit Liking and Implicit Wanting for Food}

Explicit liking and implicit wanting for food were assessed by the Leeds Food Preference Questionnaire (LFPQ). This computerised tool has been widely used in previous research [24-26] and is described in more detail elsewhere [4]. The LFPQ assesses explicit liking and implicit wanting for food using photographic stimuli. Stimuli comprise common food items categorised according to fat content (high or low) and taste (sweet or non-sweet). To measure explicit liking, participants were required to rate 'how pleasant would it be to taste some of this food now?' on a 100-mm VAS. The ratings for each food item were averaged for each 
Dalton et al.: Effect of BMI and Binge Eating on Food Reward and Energy Intake:

Further Evidence for a Binge Eating Subtype of Obesity

food type. To measure implicit wanting, a forced choice methodology was used in which an image from a given type was paired against every other image over 96 trials. Participants were required to respond according to the prompt 'which food do you most want to eat now?' as quickly and as accurately as possible, and reaction times were covertly measured. This measure provides a covert, non-verbal indication of the implicit motivational value of the foods in the task $[27,28]$. To increase statistical reliability and adjust for individual variability in overall time spent on the task, reaction times were transformed to a standardised ' $d$-score' (D-RT) using a validated algorithm [29], the procedure for which is described in greater detail elsewhere [4]. A greater implicit wanting score is indicative of a faster relative reaction time for that food type.

Test Foods

Fixed Energy Lunch Test Meal

To ensure fasted and fed conditions, the study involved consumption of a fixed energy test meal (cheese sandwich and strawberry yoghurt), individually calibrated to meet $25 \%$ of participants' daily energy requirements. Daily energy requirements were calculated using the Schofield equations [30] for basal metabolic rate multiplied by physical activity level from self-reported frequency and mode of exercise performed per week. The macronutrient content of the test meal was $32 \%$ carbohydrates; $21 \%$ protein; $47 \%$ fat. Participants ate alone in an experimental cubicle, and foods were served at the same time across conditions. Participants were required to consume all of the food that was provided to them. Food was measured to the nearest 0.1 $\mathrm{g}$, and energy values were determined using food tables and manufacturer labelling.

Ad libitum Food Intake Task

Participants were presented with six pre-weighed bowls of palatable high-fat $(\geq 40 \%)$ snack foods, chosen to be either sweet (milk chocolate, chocolate finger biscuits and cookies) or non-sweet (ready salted crisps, salted peanuts and flavoured tortilla chips). The foods were broken into irregular, bite size pieces. Participants received 60-90 g of each of each food to match for perceived quantity. Participants' acceptance of all study foods was assessed during initial screening. All foods were presented at the same time, and participants were asked to taste and rate each item on a number of sensory qualities including blandness, saltiness and sweetness using 100-mm VAS. They could rate the foods in any order and were informed they could consume as little or as much as they wanted of each item. Participants were informed that their preferences and how much they wanted to eat would be assessed at the start of the task. Foods were removed after 10 min, and each bowl was weighed so that energy intake (grams and kilocalories) could be calculated.

\section{Procedure}

Participants attended the research unit for one screening visit having fasted overnight and two lunchtime visits having fasted for at least $3.5 \mathrm{~h}$ following their normal breakfast (fig. 1). During the screening visit, participants' height, weight and waist circumference were measured. In the fasted condition, participants completed a measure of food reward (LFPQ), followed by the ad libitum food intake task. In the fed condition, the procedures were identical except that participants first consumed the fixed energy test meal to reduce levels of hunger and a period of 10 min passed following consumption of the test meal to the start of the LFPQ to allow for participants to feel full. Participants completed ratings of subjective appetite at the beginning of each test session and after each event in the procedure. On a separate day following their laboratory visits, participants completed a questionnaire booklet containing the BES. They received written and verbal debriefing and were compensated for their time before leaving the study.

\section{Data Analyses}

Data were analysed using SPSS version 18 for Windows and presented as means with standard error. Data from 4 participants ( 2 overweight or obese and 2 lean subjects) were excluded from analyses as they later confirmed they had not complied with the study fasting procedures.

The effects of hunger condition (fasted or fed state) and weight group (lean and overweight or obese) on appetite variables were assessed by mixed-model analysis of variance (ANOVA). Therefore, sensations of appetite measured at 4 time points were examined by $2 \times 2 \times 4$ mixed ANOVA. To examine the influence of trait binge eating on energy intake and food hedonic variables, ANCOVA were used with trait binge eating 
Fig. 1. Study design. Following initial screening, participants were randomly assigned to complete either the fasted or fed condition first; these had a wash-out period of 7 days. The fixed energy lunch was consumed before completing the tasks in the fed condition, and after the fasted condition. Subjective ratings of appetite were taken at the start and after each event in the procedure. Participants completed the BES on a separate day at the end of the experiment. 1LFPQ.
Dalton et al.: Effect of BMI and Binge Eating on Food Reward and Energy Intake: Further Evidence for a Binge Eating Subtype of Obesity

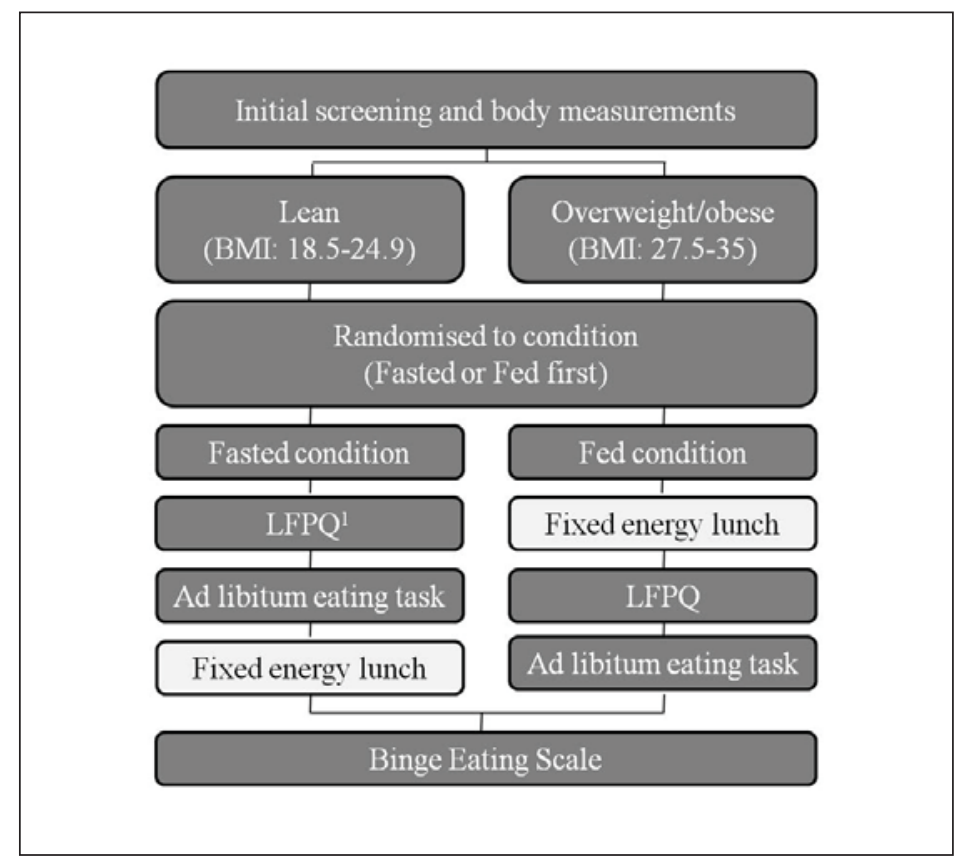

scores examined as a covariate. Therefore, food intake selection according to hunger condition, taste (nonsweet or sweet) and BMI group (lean or overweight/obese) were examined by a $2 \times 2 \times 2$ ANCOVA, and food hedonics according to hunger condition, category (high-fat savoury, low-fat savoury, high-fat sweet and low-fat sweet) and BMI group were examined by a $2 \times 2 \times 4$ ANCOVA. For analyses where significant interactions between outcome variables and trait binge eating were revealed, these effects were further examined by dividing participants into groups according to BMI (lean and overweight/obese) and binge status (bingetype or non-binge type) following a median-split of scores on the BES (table 1). Where appropriate, Greenhouse-Geisser probability levels were used to adjust for non-sphericity. Post-hoc analyses were conducted on significant interactions using the Bonferroni correction. An $\alpha$-level of 0.05 was used to determine statistical significance.

\section{Results}

\section{Subjective Appetite Sensations}

Appetite responses to the test meal were analysed to check that participants underwent measures in a fasted and a fed state. Baseline ratings of hunger, fullness, desire to eat or prospective consumption did not significantly differ in the fasted $(66.28 \pm 3.00,18.13 \pm 2.49$, $66.80 \pm 3.27$ and $61.96 \pm 2.62$, respectively) or the fed condition $(66.17 \pm 2.98,19.89 \pm 2.35$, $66.87 \pm 2.79$ and $62.91 \pm 2.94$, respectively). As expected, the test meal caused a significant reduction in hunger, with participants reporting higher levels of hunger before completing the LFPQ and the ad libitum intake task in the fasted condition (66.28 \pm 3.00 and $76.46 \pm 2.77$, respectively) than in the fed condition $(21.07 \pm 2.27$ and $31.80 \pm 3.19$, respectively) (F ( 3 , $135)=65.15, p<0.001)$. The results were similar for fullness $(p<0.001)$, desire to eat $(p<$ $0.001)$ and prospective consumption ( $<<0.001)$. There were no significant differences between overweight or obese and lean participants' self-reports of hunger, fullness, desire to eat or prospective consumption. 
Dalton et al.: Effect of BMI and Binge Eating on Food Reward and Energy Intake: Further Evidence for a Binge Eating Subtype of Obesity

Table 1. Group characteristics (mean \pm SEM) for age, BMI, fat mass, lean mass and trait binge eating

\begin{tabular}{|c|c|c|}
\hline & Binge type & Non-binge type \\
\hline \multicolumn{3}{|l|}{ Obese } \\
\hline Age, years & $25.82(2.73)$ & $25.36(2.83)$ \\
\hline BMI, $\mathrm{kg} / \mathrm{m}^{2}$ & $32(1.26)$ & $29.68(.64)$ \\
\hline Trait binge eating** & $23.1(1.39)$ & $9(1.22)$ \\
\hline Waist circumference, $\mathrm{cm}^{*}$ & $103.4(2.8)$ & $94.53(3.25)$ \\
\hline \multicolumn{3}{|l|}{ Lean } \\
\hline Age*, years & $23.3(2.33)$ & $30.23(2.22)$ \\
\hline BMI, $\mathrm{kg} / \mathrm{m}^{2}$ & $22.44(.47)$ & $21.71(.38)$ \\
\hline Trait binge eating** & $14.5(1.32)$ & $2.92(.47)$ \\
\hline Waist circumference, $\mathrm{cm}$ & $73.92(.83)$ & $73.63(1.29)$ \\
\hline \multicolumn{3}{|c|}{${ }^{*} \mathrm{p}<0.05,{ }^{* *} \mathrm{p}<0.001$} \\
\hline \multicolumn{3}{|c|}{$\mathrm{O}-\mathrm{B} \mathrm{n}=11 ; \mathrm{O}-\mathrm{NB} \mathrm{n}=12 ; \mathrm{L}-\mathrm{B} \mathrm{n}=12 ; \mathrm{L}-\mathrm{NB} \mathrm{n}=11$} \\
\hline \multicolumn{3}{|c|}{ Median cut-offs for BES: Overweight or obese 15; lean 6.} \\
\hline
\end{tabular}

\section{Ad libitum Energy Intake and Food Choice}

Participants consumed ad libitum significantly more energy in the fasted condition $(444.47 \pm 27.41)$ than in the fed condition $(349.23 \pm 23.93 ; \mathrm{t}(45)=4.395, \mathrm{p}<0.001)$. There was a main effect of food type on energy intake with participants consuming a greater proportion of sweet foods than non-sweet foods in both conditions $(F(1,45)=23.59, p<$ 0.001). There was no effect of condition on type of food consumed. Overweight or obese participants consumed more energy than lean participants in both fasted (499.21 $\pm 44.34 \mathrm{vs}$. $385.37 \pm 30.42)$ and fed $(385.76 \pm 35.92$ vs. $308.35 \pm 31.64)$ states $(F(1,44)=4.70, p<0.05)$. There were no differences in food choice (consuming sweet or non-sweet foods) between overweight or obese and lean participants.

\section{Effect of Binge Eating Subtypes on Food Intake and Appetite Variables}

Characteristics of age, BMI and waist circumference according to level of trait binge eating were compared (table 1 ). Binge eating score was significantly higher in obese binge types (O-B) and lean binge types (L-B) compared to obese non-binge types (O-NB) and lean non-binge types (L-NB), respectively. There were no differences in BMI or waist circumference between the two lean groups. Compared to O-NB, O-B had a larger waist circumference $(t(20)=2.098, p<0.05)$, but there was no difference in BMI.

\section{Subjective Appetite Sensations}

There were no significant differences between binge types in self-reported hunger, fullness, prospective consumption and desire to eat according to hunger condition $(F(9,126)$ $=1.39, \mathrm{p}>0.05 ; \mathrm{F}(9,126)=1.11, \mathrm{p}>0.05 ; \mathrm{F}(9,126)=1.19, \mathrm{p}>0.05$; and $\mathrm{F}(9,126)=1.03$, $\mathrm{p}>0.05$, respectively) (see table 2 for $\mathrm{O}-\mathrm{B}$ and $\mathrm{O}-\mathrm{NB}$ ratings). 
Fig. 2. Energy intake and intake from sweet and non-sweet food items for O-B, O-NB, L-B, and L-NB across both conditions.
Dalton et al.: Effect of BMI and Binge Eating on Food Reward and Energy Intake: Further Evidence for a Binge Eating Subtype of Obesity

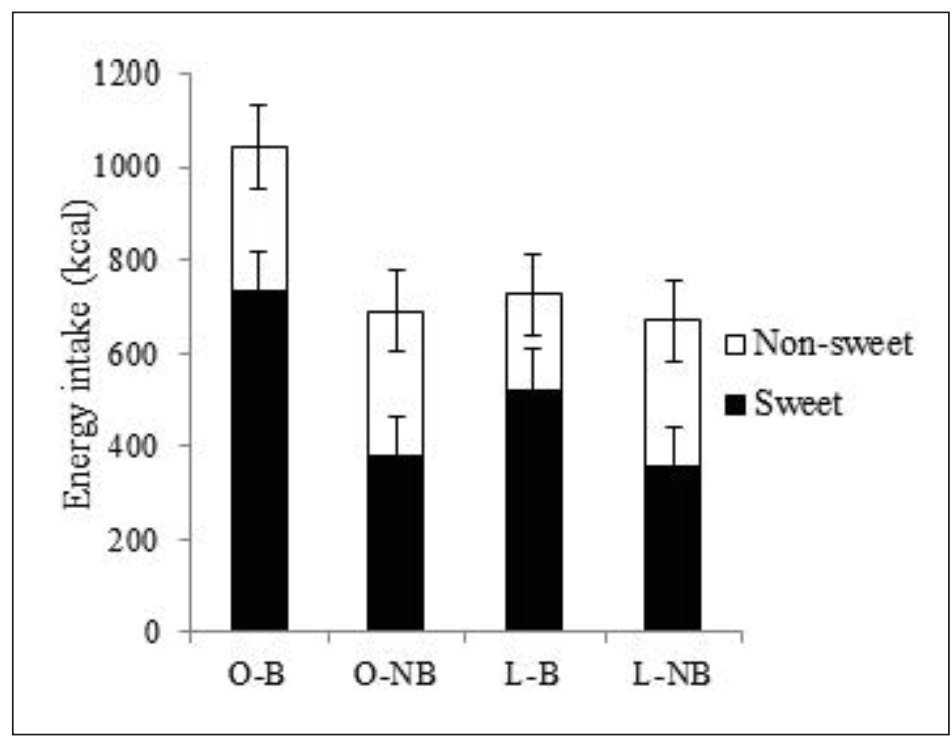

Table 2. VAS (mm) subjective appetite sensations for O-B and O-NB in the fasted and fed condition

\begin{tabular}{|c|c|c|c|c|c|c|}
\hline & \multicolumn{2}{|l|}{ VAS hunger } & \multicolumn{2}{|l|}{ VAS fullness } & \multicolumn{2}{|c|}{ VAS desire to eat } \\
\hline & O-B & $\mathrm{O}-\mathrm{NB}$ & O-B & $\mathrm{O}-\mathrm{NB}$ & O-B & $\mathrm{O}-\mathrm{NB}$ \\
\hline \multicolumn{7}{|c|}{ Fasted condition } \\
\hline Baseline & $69.25(5.86)$ & $57.55(7.12)$ & $13.17(3.28)$ & $24.82(7.26)$ & $69.42(6.50)$ & $54.65(6.66)$ \\
\hline Post task & $83.83(3.59)$ & $69.45(7.99)$ & $12.00(3.53)$ & $21.64(6.75)$ & $83.92(2.91)$ & $65.55(8.05)$ \\
\hline Post snacks & $48.00(8.72)$ & $36.36(7.17)$ & $55.83(7.21)$ & $47.27(5.75)$ & $44.42(7.75)$ & $48.73(6.42)$ \\
\hline Post lunch & $8.92(3.44)$ & $7.00(2.56)$ & $86.50(4.24)$ & $83.86(5.14)$ & $11.58(3.18)$ & $7.91(2.59)$ \\
\hline \multicolumn{7}{|c|}{ Fed condition } \\
\hline Baseline & $67.25(4.44)$ & $63.73(7.19)$ & $17.08(3.49)$ & $23.09(4.26)$ & $66.58(4.12)$ & $61.55(6.09)$ \\
\hline Post lunch & $23.67(45.25)$ & $24.91(5.88)$ & $63.17(5.04)$ & 70.55 (6.79) & $24.75(4.77)$ & $26.36(6.88)$ \\
\hline Post task & $45.25(5.81)$ & $29.09(6.94)$ & $58.42(4.97)$ & $64.82(6.18)$ & $46.25(7.56)$ & 37.09 (7.98) \\
\hline Post snacks & $16.17(5.65)$ & $8.55(2.80)$ & $78.42(6.38)$ & $80.45(5.04)$ & $23.25(6.69)$ & $12.73(2.78)$ \\
\hline
\end{tabular}

\section{Food Choice and Intake}

The influence of trait binge eating on ad libitum energy intake was analysed for overall energy intake and intake according to taste (sweet or non-sweet) (fig. 2). There was an interaction between condition and trait binge eating $(\mathrm{F}(1,43)=4.54, \mathrm{p}<0.05)$, taste and trait binge eating $(F(1,43)=12.93, p<0.001)$ and a three-way interaction between condition, taste and trait binge eating $(\mathrm{F}(1,43)=6.21, \mathrm{p}<0.02)$. To further explore these interactions and to consider the influence of trait binge eating on energy intake and food choice in lean and overweight or obese participants, participants were divided according to binge status identified using the BES to distinguish four groups: O-B, O-NB, L-B and L-NB. O-B consumed more energy overall in the fasted and fed conditions compared to the O-NB and both lean types $(F(3,42)=4.61, p<0.01)$. There was no significant difference in overall energy intake between O-NB, L-B and L-NB. 
Dalton et al.: Effect of BMI and Binge Eating on Food Reward and Energy Intake: Further Evidence for a Binge Eating Subtype of Obesity

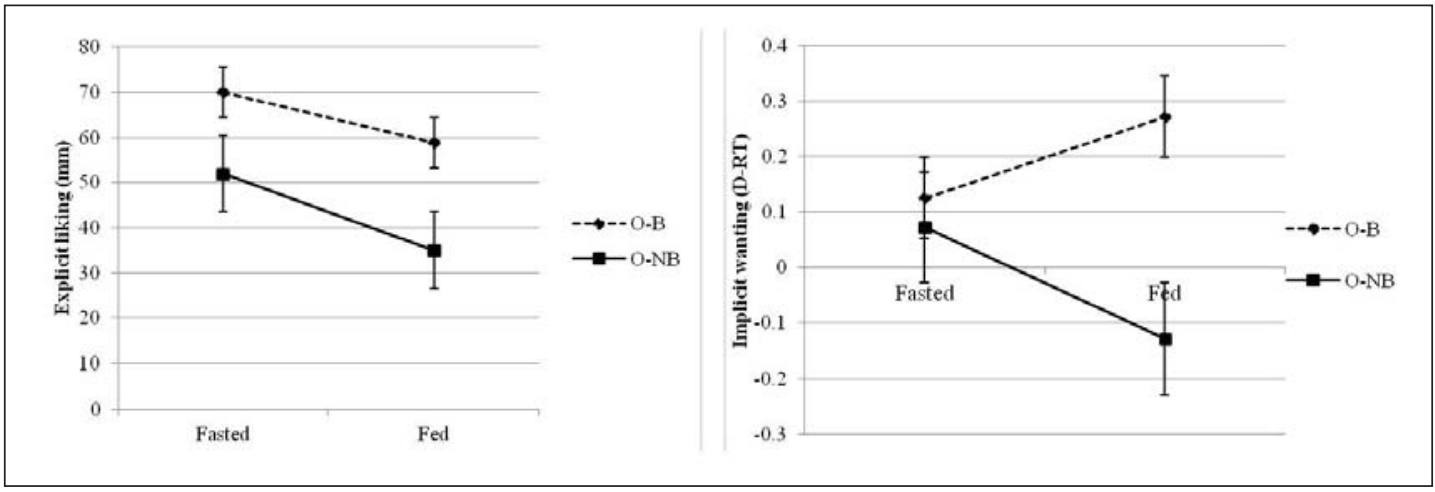

Fig. 3. left Explicit liking scores ( $\mathrm{mm}$ ) and right implicit wanting scores (D-RT) on the LFPQ for high-fat sweet foods for $\mathrm{O}-\mathrm{B}$ and $\mathrm{O}-\mathrm{NB}$ in the fasted and fed condition.

A significant binge type by food type interaction $(F(3,42)=5.86, p<0.001)$ revealed that $\mathrm{O}-\mathrm{B}$ and L-B consumed more energy from sweet foods than O-NB and L-NB, indicating that both binge types showed a preference for high-fat sweet foods. Additionally, a three-way interaction between hunger condition, food type and binge type $(F(3,42)=3.65, p<0.02)$ revealed that $\mathrm{O}-\mathrm{B}$ consumed more energy from sweet foods when fasted compared to the other subtypes.

In summary, overweight or obese participants consumed more energy ad libitum compared to lean participants in both fed and fasted states. However, when the influence of trait binge eating on energy intake and food choice was analysed, a greater intake of sweet foods was evident in O-B and L-B types, and greater overall energy intake (by approximately $30 \%$ ) was observed in the $0-B$ type.

\section{Explicit Liking and Implicit Wanting}

Analysis of explicit liking revealed that O-B had a higher explicit liking for all foods compared to $\mathrm{O}-\mathrm{NB}(\mathrm{F}(3,42)=3.23, \mathrm{p}<0.03)$. There was also a significant interaction between binge type and food type with greater explicit liking for high-fat sweet foods in O-B and L-B in both conditions $(\mathrm{F}(9,126)=2.24, \mathrm{p}<0.02)$. For both $\mathrm{O}-\mathrm{B}$ and $\mathrm{O}-\mathrm{NB}$, explicit liking for food was higher in the fasted condition compared to the fed condition $(F(1,42)=46.07, \mathrm{p}<0.001)$ (fig. 3a).

For implicit wanting, a significant interaction between binge type, food type and hunger condition on the pattern of implicit wanting was shown $(F(3,126)=2.29, p<0.02)$. When this interaction was probed, it revealed that O-B had enhanced implicit wanting for high-fat sweet foods when fed compared to O-NB; and that implicit wanting for this food type was higher in $\mathrm{O}-\mathrm{B}$ in the fed condition compared to the fasted condition, whereas the opposite pattern was present in O-NB (fig. 3b).

\section{Discussion}

The present study examined food reward and food intake during fasted and fed states according to individual differences in BMI (overweight or obese and lean) and trait binge eating (binge type or non-binge type). We hypothesised that O-B would consume more food 
Dalton et al.: Effect of BMI and Binge Eating on Food Reward and Energy Intake: Further Evidence for a Binge Eating Subtype of Obesity

and display higher liking and wanting for high-fat sweet foods than O-NB according to their degree of hunger.

The manipulation of hunger state was effective, with all participants reporting higher levels of hunger in the fasted condition compared to the fed condition. Consistent with this, participants consumed more energy in the fasted than in the fed state. The effect of hunger on explicit liking and implicit wanting was in accordance with previous research [27]. Explicit liking for all food was greater in the fasted condition compared to the fed condition, with a smaller decrease in liking for sweet compared to savoury foods. Participants' implicit wanting for non-sweet foods, but not for sweet foods, decreased following the test meal.

Consistent with previous research [1-3], we found that age-matched overweight and obese women consumed more energy compared to lean controls. Furthermore, intake was greater independent of whether participants were fasted or fed. It is important to note that these differences in intake were not due to increased levels of hunger experienced by the overweight and obese individuals, as we found no differences in self-reported appetite sensations. Indeed, one strength of the current study was that the test meal was individually calibrated to provide $25 \%$ of daily energy requirements, accounting for differences in basal energy needs of obese and lean individuals.

When the influence of trait binge eating was examined, only $0-B$ were shown to consume more energy compared to the other types. The increase in energy intake observed in 0 -B was mostly accounted for by a greater consumption of sweet foods. A similar preference for high-fat sweet foods was also evident for the L-B, although this was not associated with greater overall energy intake. These findings are consistent with previous findings in nonobese women [4]. Additionally, these findings are consistent with previous research showing that, compared to obese controls, obese individuals with binge eating disorder consume more energy in ad libitum eating tasks [31-34].

Interestingly, $\mathrm{O}-\mathrm{B}$ had a larger waist circumference than $\mathrm{O}-\mathrm{NB}$, but there was no difference in BMI. To our knowledge, a greater waist circumference in this obese subtype has not been reported previously, and therefore confirmation is needed. However, elevated waist circumference from central adiposity in 0-B may enhance their risk of obesity-related health problems $[19,35]$.

In addition to examining food intake and food choice, this study investigated the role of underlying reward processes by comparing measures of explicit liking and implicit wanting in lean and overweight or obese binge types. We found that O-B had an enhanced liking for all foods when compared to O-NB, under fed and fasted conditions. Research investigating a link between enhanced liking for foods and susceptibility to gain weight has been inconsistent to date. Blundell et al. [36] found that individuals who were overweight or obese, and habitually consumed a high-fat diet, rated food as more pleasant compared to those consuming a similar diet but were lean, but other research has reported no differences between obese and lean individuals' liking for food [3, 37]. However, the tendency to binge eat has more consistently been shown to be associated with enhanced liking. Davis et al. [14] demonstrated that individuals with binge eating disorder were more likely to have the 'gain of function' $G$ allele of the OPRM1 gene. The OPRM1 gene encodes for the mu-opioid receptor, and opioid transmission has been proposed to underpin the liking component of reward [38-40]. Furthermore, individuals with the $\mathrm{G}$ allele are proposed to have a higher liking for high-fat sweet foods [41].

In addition to enhanced liking for food in $\mathrm{O}-\mathrm{B}$, we also found evidence for dissociation between explicit liking and implicit wanting for high-fat sweet food according to hunger state. In O-B, explicit liking for high-fat sweet foods was lower in the fed condition, whereas implicit wanting for those foods was greater in the fed condition compared to the fasted condition. This apparent separation of liking and wanting was not evident in $\mathrm{O}-\mathrm{NB}$, whose wanting and 
Dalton et al.: Effect of BMI and Binge Eating on Food Reward and Energy Intake:

Further Evidence for a Binge Eating Subtype of Obesity

liking for high-fat sweet foods were both lower in the fed condition compared to fasted condition (also observed for lean participants). These findings that the binge subtype has increased implicit wanting for high-fat sweet foods are also consistent with previous research [4]. Additionally, Nasser et al. [42] reported that obese individuals with binge eating disorder (compared to healthy obese or lean subjects) showed increased motivation for food when satiated but not when hungry. This increased drive for high-fat sweet foods when satiated could be a marker of reward sensitisation in these individuals, which would convey a risk for further weight gain [43].

Although the present study carried a number of strengths, there were also some limitations to consider. First, the sample consisted of females recruited from or near a University campus; therefore, the applicability of the findings to the wider population may be limited. However, steps were taken to ensure that those recruited consisted of an equal ratio of student and non-student women. Furthermore, as the study was only conducted in females, it would be necessary to conduct further research to examine whether the findings also generalise to males. One strength of this study was that it was conducted in a controlled setting and that there was a wash-out period of at least 7 days between conditions. However, with this increased control and precision over study variables, the laboratory setting may also reduce the applicability of the findings to the free-living environment. Lastly, the sample size used in the current study poses a potential limitation to the interpretation of the results, and further replication is necessary to substantiate the novel findings.

In summary, the current study provides further evidence that trait binge eating forms part of a 'hedonic subtype' characterised by a behavioural profile of enhanced liking and implicit wanting for food. The key finding is the emergence of differences among the groups according to level of hunger. Under conditions where hunger was reduced (in the fed condition) the O-B individuals showed an enhanced implicit wanting for high-fat sweet foods compared to when fasted. Therefore, this particular condition may constitute a period of high risk for overeating in obese individuals with high binge eating scores. Identification of traits and behaviours that increase an individual's vulnerability to overeat may help to optimise treatment strategies for weight loss or prevention of weight gain. Additionally, the distinct pattern of preference for sweet foods observed for the lean binge type may have implications for the control of food intake in such individuals [e.g., 44]. The outcome demonstrates that food reward influences the control of food intake in both fed and fasted states. Additionally, the findings highlight the value of distinguishing between explicit liking and implicit wanting for food and for studying their status as risk factors for reward-driven overeating. Dissociation of liking and implicit wanting for specific food types could provide a marker for distinct patterns of eating behaviour in obesity [10].

\section{Acknowledgements}

This research was supported by Biotechnology and Biological Sciences Research Council grant: BB/ G530141/1.

\section{Disclosure Statement}

The authors declared no conflict of interest. 
Dalton et al.: Effect of BMI and Binge Eating on Food Reward and Energy Intake:

Further Evidence for a Binge Eating Subtype of Obesity

\section{References}

1 Epstein LH, Temple JL, Neaderhiser BJ, Salis RJ, Erbe RW, Leddy JJ: Food reinforcement, the dopamine $\mathrm{d}_{2}$ receptor genotype, and energy intake in obese and nonobese humans. Behav Neurosci 2007;121:877.

-2 Nijs IM, Muris P, Euser AS, Franken IH: Differences in attention to food and food intake between overweight/ obese and normal-weight females under conditions of hunger and satiety. Appetite 2010;54:243-254.

3 Saelens BE, Epstein LH: Reinforcing value of food in obese and non-obese women. Appetite 1996;27:41-50.

-4 Finlayson G, Arlotti A, Dalton M, King N, Blundell JE: Implicit wanting and explicit liking are markers for trait binge eating. A susceptible phenotype for overeating. Appetite 2011;57:722-728.

5 Guerrieri R, Nederkoorn C, Stankiewicz K, Alberts H, Geschwind N, Martijn C, Jansen A: The influence of trait and induced state impulsivity on food intake in normal-weight healthy women. Appetite 2007;49:66-73.

6 Westenhoefer J, Broeckmann P, Münch AK, Pudel V: Cognitive control of eating behavior and the disinhibition effect. Appetite 1994;23:27-41.

7 Fay SH, Finlayson G: Negative affect-induced food intake in non-dieting women is reward driven and associated with restrained-disinhibited eating subtype. Appetite 2011;56:682-688.

8 Finlayson G, Bordes I, Griffioen-Roose S, de Graaf C, Blundell JE: Susceptibility to overeating affects the impact of savory or sweet drinks on satiation, reward, and food intake in nonobese women. Journal Nutr 2012;142: 125-130.

-9 Bryant EJ, King NA, Blundell JE: Disinhibition: its effects on appetite and weight regulation. Obes Rev 2008;9: 409-419.

10 Finlayson G, Dalton M: Hedonics of food consumption: are food 'liking' and 'wanting' viable targets for appetite control in the obese? Curr Obes Rep 2012:42-49.

11 Finlayson G, Dalton M, Blundell JE: Liking versus wanting food in human appetite: Relation to craving, overconsumption and 'food addiction'; in Brownell KD, Gold MS (eds): Food and Addiction: A Comprehensive Handbook. New York, Oxford University Press, 2012, pp 220-225.

12 Berthoud HR, Morrison C: The brain, appetite, and obesity. Annu Rev Psychol 2008;59:55-92.

13 Blundell JE, Cooling J: Routes to obesity: phenotypes, food choices and activity. Br J Nutr 2000;83:33.

14 Davis C, Levitan RD, Reid C, Carter JC, Kaplan AS, Patte KA, King N, Curtis C, Kennedy JL: Dopamine for 'wanting' and opioids for 'liking': A comparison of obese adults with and without binge eating. Obesity (Silver Spring) 2009;17:1220-1225.

15 Hudson JI, Lalonde JK, Berry JM, Pindyck LJ, Bulik CM, Crow SJ, McElroy SL, Laird NM, Tsuang MT, Walsh BT: Binge-eating disorder as a distinct familial phenotype in obese individuals. Arch Gen Psychiatry 2006;63:313.

-16 Spitzer RL, Yanovski S, Wadden T, Wing R, Marcus MD, Stunkard A, Devlin M, Mitchell J, Hasin D, Horne RL: Binge eating disorder: Its further validation in a multisite study. Int J Eat Dis 1993;13:137-153.

17 Striegel-Moore RH, Rosselli F, Perrin N, DeBar L, Wilson GT, May A, Kraemer HC: Gender difference in the prevalence of eating disorder symptoms. Int J Eat Dis 2009;42:471-474.

18 Gormally J, Black S, Daston S, Rardin D: The assessment of binge eating severity among obese persons. Addict Behav 1982;7:47-55.

19 National Institutes of Health: National Heart L, Institute B: Clinical Guidelines on the Identification, Evaluation, and Treatment of Overweight and Obesity in Adults: the Evidence Report. National Institutes of Health, 1998.

20 Marcus MD, Wing RR, Hopkins J: Obese binge eaters: AFFECT, cognitions, and response to behavioral weight control. J Consult Clin Psychol 1988;56:433.

21 Freitas SR, Lopes CS, Appolinario JC, Coutinho W: The assessment of binge eating disorder in obese women: A comparison of the binge eating scale with the structured clinical interview for the DSM-IV. Eat Behav 2006; 7:282-289.

22 Timmerman GM: Binge eating scale: Further assessment of validity and reliability1. J Appl Biobehav Res 1999; $4: 1-12$.

23 Stubbs RJ, Hughes DA, Johnstone AM, Rowley E, Reid C, Elia M, Stratton R, Delargy H, King N, Blundell J: The use of visual analogue scales to assess motivation to eat in human subjects: A review of their reliability and validity with an evaluation of new hand-held computerized systems for temporal tracking of appetite ratings. Br J Nutr 2000;84:405-415.

24 Finlayson G, Caudwell P, Gibbons C, Hopkins M, King N, Blundell J: Low fat loss response after medium-term supervised exercise in obese is associated with exercise-induced increase in food reward. J Obes 2011;2011

25 Griffioen-Roose S, Finlayson G, Mars M, Blundell JE, de Graaf C: Measuring food reward and the transfer effect of sensory specific satiety. Appetite 2010;55:648-655.

26 Verschoor E, Finlayson G, Blundell J, Markus CR, King NA: Effects of an acute $\alpha$-lactalbumin manipulation on mood and food hedonics in high-and low-trait anxiety individuals. Br J Nutr 2010;104:595-602.

-27 Finlayson G, King N, Blundell J: The role of implicit wanting in relation to explicit liking and wanting for food: Implications for appetite control. Appetite 2008;50:120-127.

28 Finlayson G, Bryant E, Blundell JE, King NA: Acute compensatory eating following exercise is associated with implicit hedonic wanting for food. Physiol Behav 2009;97:62-67.

29 Greenwald AG, Nosek BA, Banaji MR: Understanding and using the implicit association test: I. An improved scoring algorithm. J Pers Soc Psychol 2003;85:197.

-30 Schofield W: Predicting basal metabolic rate, new standards and review of previous work. Hum Nutr Clin Nutr 1985;39:5-41. 
Dalton et al.: Effect of BMI and Binge Eating on Food Reward and Energy Intake: Further Evidence for a Binge Eating Subtype of Obesity

31 Geliebter A, Hassid G, Hashim SA: Test meal intake in obese binge eaters in relation to mood and gender. Int J Eat Dis 2001;29:488-494.

-32 Goldfein JA, Walsh BT, Devlin MJ, Lachaussée JL, Kissileff HR: Eating behavior in binge eating disorder. Int J Eat Dis 1993;14:427-431.

33 Latner JD, Rosewall JK, Chisholm AM: Food volume effects on intake and appetite in women with binge-eating disorder and weight-matched controls. Int J Eat Dis 2009;42:68-75.

34 Yanovski SZ, Leet M, Yanovski JA, Flood M, Gold PW, Kissileff HR, Walsh BT: Food selection and intake of obese women with binge-eating disorder. The Am jJ Clin Nutr 1992;56:975-980.

-35 Zhu SK, Wang ZM, Heshka S, Heo M, Faith MS, Heymsfield SB: Waist circumference and obesity-associated risk factors among whites in the third national health and nutrition examination survey: clinical action thresholds. Am J Clin Nutr 2002;76:743.

-36 Blundell JE, Stubbs RJ, Golding C, Croden F, Alam R, Whybrow S, Le Noury J, Lawton CL: Resistance and susceptibility to weight gain: individual variability in response to a high-fat diet. Physiol Behav 2005;86:614-622.

-37 Cox D, Perry L, Moore P, Vallis L, Mela D: Sensory and hedonic associations with macronutrient and energy intakes of lean and obese consumers. Int J Obes 1999;23:403-410.

-38 Berridge KC: Food reward: BRAIN substrates of wanting and liking. Neurosci Biobehav Rev 1996;20:1-25.

39 Berridge KC, Robinson TE: Parsing reward. Trends Neurosci 2003;26:507-513.

40 Pecina S, Smith KS, Berridge KC: Hedonic hot spots in the brain. Neuroscientist 2006;12:500-511.

-41 Davis C, Zai C, Levitan RD, Kaplan AS, Carter JC, Reid-Westoby C, Curtis C, Wight K, Kennedy JL: Opiates, overeating and obesity: a psychogenetic analysis. Int J Obes (Lond) 2011;35:1347-1354.

42 Nasser JA, Evans SM, Geliebter A, Pi-Sunyer FX, Foltin RW: Use of an operant task to estimate food reinforcement in adult humans with and without bed. Obesity 2008;16:1816-1820.

-43 Temple JL, Bulkley AM, Badawy RL, Krause N, McCann S, Epstein LH: Differential effects of daily snack food intake on the reinforcing value of food in obese and nonobese women. Am J Clin Nutr 2009;90:304-313.

44 Blundell J, Finlayson G: Role du sucre dans le controle de l'appetit. Cah Nutr Diet 2008;43:2. 